\section{Clinical Impact of Probiotics among Patients Receiving Antibiotics with a High Risk of Acquiring Clostridium difficile Infection}

\section{Abstract}

Importance: Despite recommendations not to routinely use probiotics for primary prevention of Clostridium difficile infection (CDI) from Updated Practice Guidelines for CDI in 2010 by the Society for Healthcare Epidemiology of America and the Infectious Diseases Society of America, the use of probiotics remains common among prescribers and patients. To our knowledge, there has been no analysis conducted to evaluate the impact of probiotics on the primary prevention of CDI among patients receiving antibiotics known to be associated with an increased risk of CDI.

Objective: To determine whether patients who receive "high-risk" antibiotics along with probiotics are at a decreased risk of developing CDI.

Design, setting, and participants: This was a retrospective cohort study including adult patients admitted to Yale New Haven Hospital, Saint Raphael Campus, between July 1, 2010 and December 31, 2010. Patients were excluded if they did not receive high-risk antibiotic(s) for more than 5 days nor had a history of CDI.

Interventions: Eligible patients were then analyzed based on whether they had received concomitant use of probiotics or antibiotics alone.

Main outcome(s) and measure(s): The primary outcome of the study was the development of $\mathrm{CDI}$ within 90 days of high-risk antibiotic use.

Results: A total of 389 patients were included in the study. CDI occurred in $8.4 \%$ $(12 / 143)$ of patients who received concomitant probiotics as opposed to $3.3 \%$ $(8 / 246)$ that had $C D I$ and received antibiotics alone with relative risk (RR) of $2.58(95 \% \mathrm{Cl}: 1.08,6.16 ; \mathrm{p}=0.033)$. A chi-square analysis identified statistically significant differences in age $(p<0.0001)$ and proton-pump inhibitor (PPI) utilization $(p=0.0088)$, but the imbalance between patients with and without probiotics was removed after adjusting for the propensity score $(p=0.1141)$.

Conclusion and relevance: We found that use of antibiotics along with probiotics had a significantly higher incidence of CDI than those who did not receive probiotics. Our findings based on adjusted odds ratio do not support the recommendation of the routine use of probiotics for the prevention of CDI. Other strategies such as eliminating the unnecessary use of PPI should be applied to prevent CDI.

\section{Justine E Dickson", Whitney Y Hung ${ }^{2}$ and Tai-Tsang Chen ${ }^{3-5}$}

\author{
1 Boston Medical Center, Boston, MA, USA \\ 2 University Medical Center of Princeton, \\ Plainsboro, NJ, USA \\ 3 Columbia University, Mailman School of \\ Public Health, New York, NY, USA \\ 4 Marjorie Golden, MD, FACP, USA \\ 5 Yale University School of Medicine, New \\ Haven, CT, USA
}

Corresponding author:

Whitney Y Hung

\section{झ whitney0522@gmail.com}

University Medical Center of Princeton, One Plainsboro Road, Plainsboro, NJ 08536, USA.

Tel: (609)853-6114

Citation: Dickson JE, Hung WY, Chen TT. Clinical Impact of Probiotics among Patients Receiving Antibiotics with a High Risk of Acquiring Clostridium difficile Infection. Int J Dig Dis. 2016, 2:1.

Received: January 05, 2016; Accepted: February 03, 2016; Published: February 13, 2016

\section{Background}

Clostridium difficile is the most commonly reported nosocomial pathogen, accounting for $12 \%$ of healthcare-associated infections [1]. It is considered an avoidable cause of morbidity and mortality among hospitalized patients [2]. Incidence and severity of Clostridium difficile infection (CDI) have risen substantially since the early 1990s. In the United States, patients who develop CDI in the hospital tend to have increased length of stay (LOS) with an 
estimated total cost for treatment exceeding $\$ 1.1$ billion annually [3]. Centers for Medicare and Medicaid Services (CMS) have targeted CDI as one of its new quality measures for FY2017 [4].

Several approaches have been deployed to decrease rates of $\mathrm{CDI}$ including promoting proper hand hygiene, developing antimicrobial stewardship programs to control the unnecessary use of antimicrobial agents, minimizing use of drugs known to increase risk of CDI such as proton-pump inhibitors (PPI), [5] and improving compliance with isolation precautions. Administering probiotics concomitantly with antibiotic treatment has been considered as a potential strategy for preventing CDI. There have been a variety of studies that investigated the potential benefits of using probiotics for either primary or secondary $\mathrm{CDI}$ prophylaxis; however, the findings were inconclusive $[6,7]$. According to the Clinical Practice Guidelines for CDI in Adults: 2010 Update by the Society for Healthcare Epidemiology of America (SHEA) and the Infectious Diseases Society of America (IDSA), the administration of probiotics is not recommended for the prevention of primary $C D$ due to limited data and conflicting results. Nevertheless, the use of probiotics remains common among prescribers and patients in order to avoid CDI [8].

In addition to certain host factors associated with CDI, it was found that antibiotics may inherently have different risks of causing CDI [9]. These high-risk antibiotics include carbapenems (imipenem-cilastin and meropenem), clindamycin, and third generation cephalosporins (ceftazidime, cefpodoxime, and ceftriaxone). Fluoroquinolones (FQs; ciprofloxacin, levofloxacin, and moxifloxacin) have emerged as other potential offenders for hospital acquired CDI [10]. The similar relative risks of specific antibiotics were found in community-associated CDI [11]. To our knowledge, there has been no analysis conducted to evaluate the impact of probiotics on the primary prevention of CDI among patients receiving such "high-risk" antibiotics. Our objective was to evaluate the role of probiotics for primary prevention of $C D I$ in adult patients receiving antibiotics known to be associated with an increased risk of CDI.

\section{Methods}

This was a retrospective cohort study evaluating the impact of probiotic use on the primary prevention of CDI in adult patients ( $\geq$ 18 years old) admitted to Yale New Haven Hospital, Saint Raphael Campus, a 551-bed teaching hospital, between July 1, 2010 and December 31, 2010. Patients who did not receive any high-risk antibiotic for more than 5 days nor had a history of CDI were excluded from the review. The Computerized Physician Order Entry (CPOE) system, QuadraMed ${ }^{\circledR}$, was used to collect patient information. Patients were identified for study inclusion if they had a Charge Description Master (CDM) number for the following highrisk antibiotics: ceftriaxone, ciprofloxacin, clindamycin, imipenemcilastin, levofloxacin, or meropenem.

Patients who had a CDM number for one of the high-risk antibiotics were screened by electronic chart review to determine (1) if the minimum duration of antibiotic therapy was achieved ( $\geq 5$ days) and ( 2 ) if the patient had prior history of CDI. Eligible patients were then analyzed based on whether they had received concomitant use of probiotics or antibiotics alone.

The following patient-level information was abstracted: age, gender, race, presence of comorbid conditions (history of human immunodeficiency virus infection/acquired immune deficiency syndrome (HIV/AIDS), underlying malignancy, gastrointestinal disease, and cardiovascular disease). Information was also gathered on high-risk antibiotic(s) use, results of $C$. difficile diagnostic tests from the institution's laboratory reports during their inpatient stay as well as 90 days post antibiotic therapy $[12,13]$ type and duration of probiotic use, use of a PPI, and baseline serum creatinine and albumin levels. Pantoprazole was the formulary PPI and Lactobacillus GG (Culturelle ${ }^{\circledR}$ ) as well as Saccharomyces boulardii (Florastor ${ }^{\circledR}$ ) was the formulary probiotics agents available during the study period.

At the time of the study, the microbiology laboratory utilized an enzyme immunoassay (EIA) to detect glutamate dehydrogenase $(\mathrm{GDH})$ for $C$. difficile antigen and toxin $A$ and $B$ production ( $C$. Diff Quik Check Complete ${ }^{\circledR}$, TECHLAB, Inc.). Submitted stools were rejected if well formed. If both $C$. difficile antigen and toxin were positive, the test was considered positive and treatment of CDI was indicated. Polymerase chain reaction (PCR) (Xpert ${ }^{\circledast} C$. difficile, Cepheid, Inc.) was used as the confirmatory test if there was discordance between toxin and antigen testing (i.e., antigen positive, toxin negative). If the result of PCR was negative, the patient was deemed to be free of CDI.

The primary outcome of the study was the development of CDI within 90 days of high-risk antibiotic use. This research proposal was approved by our institution's Investigational Review Board (IRB).

\section{Statistical Analysis}

Discrete demographic variables were tabulated by the treatment arm (i.e., probiotic use vs. antibiotics alone), and by the frequency and the proportion of patients falling into each category. Percentages given in the tables will be rounded and, therefore, may not always sum up to $100 \%$. Continuous variables were summarized by the treatment arm using the mean and range (min-max). All analyses were conducted using all available data from patients who met the study inclusion criteria.

The magnitude of the difference in $\mathrm{CDI}$ incidence rates between two treatment arms was captured using relative risk along its 95\% confidence interval. A sensitivity analysis adjusting for the propensity score was conducted to control for the selection bias of the probiotic use. Propensity score was defined as the conditional probability of receiving probiotics given a set of key risk factors. The propensity score was estimated for each patient using a multiple logistic regression model with stepwise selection to evaluate the covariates of the PPI use, gender, age, levofloxacin, ceftriaxone, ciprofloxacin, clindamycin, gastrointestinal disorder, history of malignancy, serum creatinine, average level of albumin and age. The stepwise selection procedure removed any insignificant variables from the model before adding a significant one to the model. The propensity scores were then ranked and grouped into five strata. An adjusted relative risk was estimated using a Cochran-Mantel-Haenszel (CMH) statistic with associated $95 \% \mathrm{Cl}$, stratified by five propensity score categories. All analyses were carried out using SAS software Version 9.3 (SAS Institute, Cary, NC, USA).

\section{Results}

In this study, 2,273 adult inpatients received at least one dose of a high-risk antibiotic from July 1, 2010 to December 31, 2010. 
After excluding patients who had received fewer than 5 days of high-risk antibiotic therapy as well as those with a history of CDI, a total of 389 patients met the study inclusion criteria. Of these, 143 patients received probiotics. In this study, $62.7 \%$ (244 out of 389) of the patients were female. Baseline characteristics were balanced between patients with and without probiotics, with the exception of age and the use of PPI (Table 1). Patients who received probiotics concomitantly with high-risk antibiotics were on average older than those who were given antibiotics alone (80.3 vs. 73.34 years, respectively $(p<0.05)$. In addition, a significantly higher proportion of patients in probiotic group had concurrent use of PPI than patients in antibiotic alone group (57.3\% vs. $43.5 \%, p<0.05$ ).

Probiotics were prescribed less frequently for patients with malignancy (20.98\% vs. $27.24 \%$ ), but more often for patients with other underlying gastrointestinal disorder (24.48\% vs. $22.76 \%)$. The percentages of patients with baseline coronary artery disease (CAD) were similar between the group utilizing probiotics and that with antibiotic alone (36.11\% vs. $34.29 \%)$.

Overall exposure to FQs, ceftriaxone, and clindamycin was similar in the 2 groups. There was statistically significantly higher usage of levofloxacin in probiotic group and higher usage of ciprofloxacin in antibiotic alone group (Table 2). No patient who received carbapenems during the study time period was included in either group. A total of nine patients received more than one high-risk antibiotic for 5 days or more. The average duration of antibiotic use was slightly lower in the probiotics group (7.3 days vs. 7.7 days) with 63.27 to $65.28 \%$ of patients receiving antibiotic treatment for 5-7 days (Figure 1). The majority of patients in the probiotic group received Lactobacillus GG, compared to Saccharomyces boulardii ( $82.52 \%$ vs. $23.07 \%) ; 4.9 \%$ of patients received both probiotics. Duration of either Lactobacillus GG or Saccharomyces ranged from 1 day to 35 days, with most patients (31.94\%) receiving 5-7 days of probiotic therapy.

Overall, CDI was detected in 20 out of 389 (5.1\%) patients within 90 days of high-risk antibiotic use. CDI occurred in $8.4 \%(12 / 143)$ of patients who received concomitant probiotics as opposed to $3.3 \%(8 / 246)$ that had CDI and received antibiotics alone with relative risk (RR) of 2.58 (95\% Cl: 1.08, 6.16; $p=0.033$ ) (Table 3). Between these two groups, a chi-square analysis identified statistically significant differences in age $(p<0.0001)$ and $P P I$ utilization $(p=0.0088)$. Using a multiple regression analysis to account for the difference in age between the two groups, the RR was trending towards significance at $2.55(95 \% \mathrm{Cl}: 0.99,6.54)$. A similar regression analysis was also conducted in order to account for statistically significant difference in PPI utilization between the groups, resulting in a similar RR of 2.46 (95\% Cl: $0.97,6.23)$. When both age and use of PPI were included in the model, the analysis yielded a RR of $2.32(95 \% \mathrm{Cl}: 0.90,5.96)$.

When all demographic and baseline clinical characteristics were included in the multiple logistic regression model, four covariates were identified to be different between two groups at the entry criterion of 0.1 in the stepwise selection procedure: age, use of PPI, and use of both levofloxacin and ciprofloxacin. The imbalance between patients with and without probiotics was removed after adjusting for the propensity score (Table 4 ). The sensitivity analysis adjusting for the propensity score yielded a non-significant RR of 2.53 (95\% Cl: $0.93,6.94 ; p=0.1141)$.

\section{Discussion}

The emergence of CDI has compelled clinicians and researchers to identify any potential intervention to decrease the incidence of both hospital as well as community associated CDI. Increasing attention has been paid to the colonic microbiome with resultant attempts to repopulate the gut with normal flora. Probiotics are live microorganisms which are thought to be able to restore the gut flora disrupted by antibiotic use, maintaining normal carbohydrate metabolism and possibly competitively inhibiting growth of pathogens [12]. Studies have demonstrated significant benefit of utilizing different strains of probiotics in the reduction of antibiotic-associated diarrhea (AAD) in pediatric population with no major impact on CDI [13-16]. Our patient population differs from those included in many of these studies. No pediatric patients were included in this investigation; all of our study patients were adults recruited from an urban US hospital. Isolation of $C$. difficile toxin from stools of children may not have the same implications as in adults, and studies have suggested that detection of $C$. difficile toxin in the stools of children may not even necessarily be the cause of diarrhea [17]. In addition, our study looked only at patients with CDI, not antibiotic associated diarrhea and specifically focused on those receiving high risk antibiotics where potential benefits of probiotic prophylaxis should have been even more compelling.

The testing methods of $C D I$ have evolved in the past decade. Our study included utilized PCR testing for detection of $\mathrm{C}$. difficile toxin. This method was not developed until 2003 [18]. Utilizing this technology, we may have detected more cases of CDI than prior studies.

Overall, there is a lack of scientific evidence showing efficacy for the use of probiotics for primary prevention of CDI. Owing to low cost and rare occurrence of important adverse effects, probiotics are frequently prescribed and utilized by patients, especially for patients who received high-risk antibiotics [7]. To our knowledge, this is the first study designed to evaluate the role of probiotics in primary CDI prophylaxis for patients receiving antibiotics with an increased risk of CDI. Given the focus of our study was on patients who receive high risk antibiotics, if there is no benefit associated with high risk antibiotics and probiotics, it is likely that probiotics may not provide significant impact on low risk antibiotics.

We found that utilization of high-risk antibiotics (ceftriaxone, ciprofloxacin, clindamycin, or levofloxacin) is associated with an increased risk of CDI, which was not decreased by concomitant use of probiotics. Documented risk factors based on published studies for CDI including advanced age, the use of PPI, inflammatory bowel disease, organ transplantation, chemotherapy, chronic kidney disease, immunodeficiency, as well as low albumin level were evaluated [19]. Differences were minimized after potential biases (i.e., older age, the use of PPI, levofloxacin, and ciprofloxacin) were adjusted based on statistic model.

There were no baseline characteristics other than age, use of PPIs, and the use of levofloxacin versus ciprofloxacin that could be identified between the two study groups as increasing risk for CDI. Patients who received both antibiotic and probiotic were significantly older than those who received antibiotics alone 80.41 vs. 73.3 years (Table 1). The overall use of FQs (ciprofloxacin and levofloxacin) was similar between the two groups. Although levofloxacin was utilized more in the group with concomitant use 
Table 1. Demographic and clinical characteristics of patients who received a minimum of five days of treatment with a high-risk antibiotic, with or without concomitant probiotic use.

\begin{tabular}{|c|c|c|c|c|c|}
\hline $\begin{array}{l}\text { Characteristic } \\
\text { Sex }\end{array}$ & \multicolumn{2}{|c|}{$\begin{array}{l}\text { Probiotic Group } \\
\qquad(N=143)\end{array}$} & \multicolumn{2}{|c|}{$\begin{array}{l}\text { Antibiotic Alone Group } \\
\qquad(N=246)\end{array}$} & $\begin{array}{c}\text { P-value } \\
0.25\end{array}$ \\
\hline Male, n (\%) & 48 & $33.57 \%$ & 97 & $39.43 \%$ & \\
\hline Female, n (\%) & 95 & $66.43 \%$ & 149 & $60.57 \%$ & \\
\hline Age Group & & & & & 0.0001 \\
\hline$<45$ years, $\mathrm{n}(\%)$ & 1 & $0.70 \%$ & 12 & $4.88 \%$ & \\
\hline 45 to $<65$ years, $n(\%)$ & 19 & $13.29 \%$ & 50 & $20.33 \%$ & \\
\hline 65 to $<75$ years, $n(\%)$ & 17 & $11.89 \%$ & 54 & $21.95 \%$ & \\
\hline 75 to $<85$ years, $n(\%)$ & 49 & $34.27 \%$ & 60 & $24.39 \%$ & \\
\hline$\geq 85$ years, $n(\%)$ & 57 & $39.86 \%$ & 70 & $28.46 \%$ & \\
\hline Age, mean, years (range) & 80.41 & $(40-103)$ & 73.30 & $(16-104)$ & \\
\hline Average $\mathrm{SCr}, \mathrm{mg} / \mathrm{dL}$ (range) & 1.28 & $(0.4-8.5)$ & 1.25 & $(0.2-15.9)$ & 0.80 \\
\hline Average Albumin level, g/dL (range) & 3.38 & $(1-4.7)$ & 3.39 & $(1.9-4.8)$ & 0.89 \\
\hline Patients with level, $\mathrm{n}(\%)$ & 127 & $88.81 \%$ & 205 & $83.33 \%$ & \\
\hline Days of Antibiotic Therapy & & & & & 0.48 \\
\hline 5 to 7 days, $\mathrm{n}(\%)$ & 94 & $65.28 \%$ & 155 & $63.27 \%$ & \\
\hline 8 to 10 days, $\mathrm{n}(\%)$ & 37 & $25.69 \%$ & 61 & $24.90 \%$ & \\
\hline$>10$ days, $\mathrm{n}(\%)$ & 16 & $11.11 \%$ & 35 & $14.29 \%$ & \\
\hline Average duration of therapy, days (range) & 7.29 & $(5-25)$ & 7.72 & $(5-37)$ & \\
\hline \multicolumn{6}{|l|}{ Probiotic Use } \\
\hline Lactobacillus GG, n (\%) & 118 & $81.94 \%$ & - & - & \\
\hline Saccharomyces boulardii, n (\%) & 33 & $22.92 \%$ & - & - & \\
\hline Multiple Probiotic Therapy, n (\%) & 7 & $4.86 \%$ & - & - & \\
\hline \multicolumn{6}{|l|}{ Days of Probiotic Use } \\
\hline$<5$ days, $\mathrm{n}(\%)$ & 41 & $28.47 \%$ & - & - & \\
\hline 5 to 7 days, $\mathrm{n}(\%)$ & 46 & $31.94 \%$ & - & - & \\
\hline 8 to 10 days, $\mathrm{n}(\%)$ & 36 & $25.00 \%$ & - & - & \\
\hline$>10$ days, $\mathrm{n}(\%)$ & 28 & $19.44 \%$ & - & - & \\
\hline Average duration of therapy, days (range) & 8.35 & $(1-35)$ & - & - & \\
\hline History of HIV/AIDS, n (\%) & 0 & $0.00 \%$ & 1 & $0.41 \%$ & $\mathrm{~N} / \mathrm{A}$ \\
\hline History of malignancy, n (\%) & 30 & $20.98 \%$ & 67 & $27.24 \%$ & 0.17 \\
\hline Gastrointestinal Disorder, n (\%) & 35 & $24.48 \%$ & 56 & $22.76 \%$ & 0.70 \\
\hline Celiac Disease & 0 & $0.00 \%$ & 1 & $0.41 \%$ & \\
\hline Chronic Diarrhea & 0 & $0.00 \%$ & 2 & $0.82 \%$ & \\
\hline Colectomy & 1 & $0.69 \%$ & 0 & $0.00 \%$ & \\
\hline Colon Cancer & 2 & $1.39 \%$ & 1 & $0.41 \%$ & \\
\hline Colonic Polyps & 1 & $0.69 \%$ & 0 & $0.00 \%$ & \\
\hline Colostomy & 1 & $0.69 \%$ & 1 & $0.41 \%$ & \\
\hline Crohns Disease & 0 & $0.00 \%$ & 2 & $0.82 \%$ & \\
\hline Diverticulitis & 7 & $4.86 \%$ & 8 & $3.27 \%$ & \\
\hline Feeding Tube & 1 & $0.69 \%$ & 0 & $0.00 \%$ & \\
\hline Gastric Ulcer & 0 & $0.00 \%$ & 1 & $0.41 \%$ & \\
\hline Gastritis & 1 & $0.69 \%$ & 3 & $1.22 \%$ & \\
\hline Gastroperesis & 0 & $0.00 \%$ & 3 & $1.22 \%$ & \\
\hline Gastroesophageal Reflux Disease & 17 & $11.81 \%$ & 27 & $11.02 \%$ & \\
\hline H. pylori & 0 & $0.00 \%$ & 2 & $0.82 \%$ & \\
\hline Ileocolectomy & 0 & $0.00 \%$ & 1 & $0.41 \%$ & \\
\hline Ischemic Colitis & 1 & $0.69 \%$ & 3 & $1.22 \%$ & \\
\hline Peptic Ulcer Disease & 3 & $2.08 \%$ & 6 & $2.45 \%$ & \\
\hline Short Gut & 0 & $0.00 \%$ & 1 & $0.41 \%$ & \\
\hline Ulcerative Colitis & 3 & $2.08 \%$ & 1 & $0.41 \%$ & \\
\hline Coronary Artery Disease, $\mathrm{n}(\%)$ & 52 & $36.11 \%$ & 84 & $34.29 \%$ & 0.66 \\
\hline Use of PPI*, n (\%) & 82 & $57.34 \%$ & 107 & $43.50 \%$ & 0.01 \\
\hline
\end{tabular}

4 
Table 2. The use of antibiotic therapy.

\begin{tabular}{|c|c|c|c|c|c|}
\hline \multirow[b]{2}{*}{ Levofloxacin, n (\%) } & \multicolumn{2}{|c|}{ Probiotic Group } & \multicolumn{2}{|c|}{ Antibiotic Alone Group } & \multirow{2}{*}{$\begin{array}{c}\text { P-value } \\
0.03\end{array}$} \\
\hline & 88 & $61.54 \%$ & 123 & $50.00 \%$ & \\
\hline Ciprofloxacin, n (\%) & 34 & $23.78 \%$ & 91 & $36.99 \%$ & 0.01 \\
\hline Ceftriaxone, n (\%) & 19 & $13.29 \%$ & 25 & $10.16 \%$ & 0.35 \\
\hline Clindamycin, n (\%) & 5 & $3.50 \%$ & 12 & $4.88 \%$ & 0.70 \\
\hline Meropenem, n (\%) & 0 & $0.00 \%$ & 0 & $0.00 \%$ & N/A \\
\hline Imipenem-Cilastin, n (\%) & 0 & $0.00 \%$ & 0 & $0.00 \%$ & N/A \\
\hline Multiple Drug Therapy, n (\%) & 3 & $2.08 \%$ & 6 & $2.45 \%$ & 0.83 \\
\hline
\end{tabular}

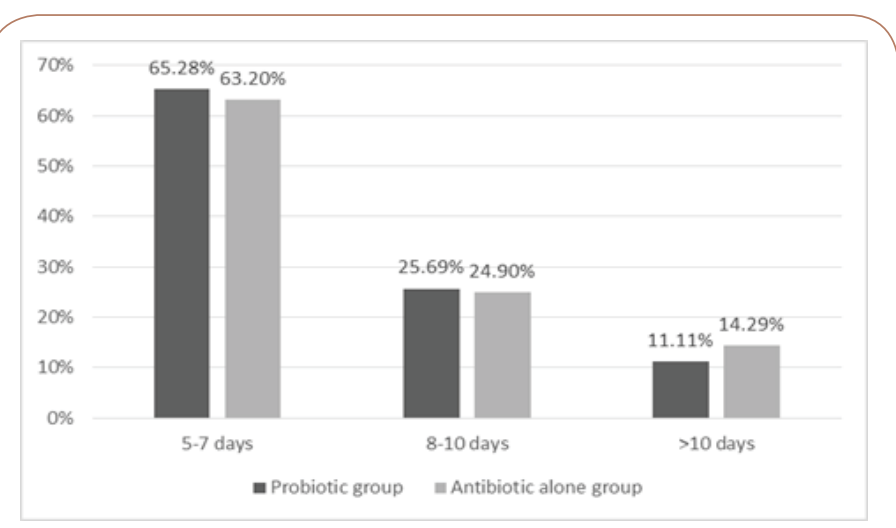

Figure 1 Duration of antibiotic therapy.

of probiotics, in vitro studies have not shown that levofloxacin promoted growth of $C$. difficile or increased toxin production more than ciprofloxacin. Moxifloxacin, on the other hand, with enhanced anti-anaerobic activity may have a greater propensity to induce CDI than ciprofloxacin and levofloxacin [20]

The U.S. Food and Drug Administration (FDA) released a drug safety communication in 2012 about PPI and their association with CDI [5]. PPIs are known to increase gastric $\mathrm{pH}$ levels via selectively and irreversibly inhibiting gastric hydrogen/potassium adenosine triphosphatase $(\mathrm{H}+/ \mathrm{K}+-$ exchanging ATPase). This enzyme is part of the 'proton pump' that performs the ultimate step in the acid secretory process [21]. One of the mechanism of PPI associated $\mathrm{CDI}$ is that PPIs raise the $\mathrm{pH}$ of gastric contents, thus interfering with the ability of gastric acid to kill $C$. difficile spores, leading to germination and outgrowth of spores [22].

PPIs quickly became the third most used medication in the United States shortly after the products became available over the counter [23]. Growing evidences suggests that these medications are being used in the absence of clear indications [24]. Although the proper use of PPI was not evaluated in the current study, only $11 \%$ of patients in both groups had history of GERD while $43-57 \%$ of patients were receiving PPI. Reducing the use of these agents should be considered as a control strategy for CDI in the future.

Patients with hypoalbuminemia (a serum albumin level of $2.5 \mathrm{~g} /$ $\mathrm{dL}-3.0 \mathrm{~g} / \mathrm{dL})[25,26]$ have been shown to be at an increased risk of contracting severe $\mathrm{CDI}$, as well as those with a serum creatinine of $2.26 \mathrm{~g} / \mathrm{dL}$ or greater. [27] Looking at patient demographics, there was no significant difference between groups, thereby rejecting the null hypothesis that there were inherent differences in risk to develop CDI between the two groups.

Although differences were minimized after potential biases (i.e., older age, the use of PPI, levofloxacin, and ciprofloxacin) and were adjusted based on a statistic model, it did raise an interesting question as to whether there are any interactions among PPIs, probiotics, and CDI. In general, probiotics are resistant to gastric acidity and reach high concentration level in gastrointestinal tract in order to restore the gut flora. It has been hypothesized that by creating a more neutral gastric $\mathrm{pH}$, PPIs allow ingested bacteria to survive and negatively impact normal gut microbiome [28]. They may also impact effects of probiotics, but more research is needed to understand this process.

Probiotics should not be considered as risk-free agents, since cases of probiotic-associated endocarditis as well as bacteremia have been reported in the literature [29]. These types of infections indicate the ability for probiotics to translocate. Translocation of bacteria is caused by a defective intestinal barrier, gut prematurity, or immunosuppression, which results in the transfer of bacteria to other organs. This transfer of bacteria may further lead to bacteremia, septicemia, and multi-organ failure [30]. Lactobacillus rhamnosus (GG) was found to have the second highest rate of isolation (22.9\%) in a study of 241 cases of infection caused by probiotics. Mortality rates from these Lactobacillus-associated infections was found to be $29.1 \%(P=0.004)$ in a case series study [29]. These infections have been associated with patients who have received recent surgery, organ transplant, or have diabetes mellitus, AIDS, or cancer [30].

At the time of the study, probiotics were prescribed based on each provider's clinical judgment without standardized protocol resulting approximately $\$ 10,000$ per year on probiotics. The acquisition costs may be close to $\$ 30,000$ a year if the decision was made to give all patients receiving antibiotics with an increased risk of $\mathrm{CDI}[30,31]$. This will result in significant expense without a clear benefit based on the results of present study.

All probiotics were completely removed from our hospital formulary in June 2013. We did not observe an increase of CDI in 2014. In fact, the rate of CDI decreased from 9.6 patients $/ 10,000$ patient-days in 2013 to 7.14 patients/10,000 patient-day in 2014, although the impact of other initiatives and formulary changes cannot be ruled out.

Our study had several limitations. This review was conducted at a single center with retrospective study design. In comparing the two groups, there were $72 \%$ more patients in the group receiving antibiotics alone than the group receiving both antibiotic and probiotic. Although propensity score approach was implemented, the potential selection bias cannot be eliminated completely. Lactobacillus G.G. and Saccharamyces boulardii were the only 2 probiotic products included in the study. In addition, we were only able to identify patient with $C D I$ if the 
Table 3. Incidence of $C D I$ with a minimum of five days of high-risk antibiotic therapy, with or without concomitant probiotic use.

$\begin{array}{lcc}\text { Characteristic } & \begin{array}{c}\text { Probiotic } \\ \text { Group } \\ (\mathrm{N}=143)\end{array} & \begin{array}{c}\text { Antibiotic } \\ \text { Alone Group } \\ (\mathrm{N}=246)\end{array} \\ \text { Clostridium difficile detected, } \mathrm{n}(\%) & 12(8.4) & 8(3.3) \\ \text { CDI while using PPI*, } \mathrm{n}(\%) & 10(83.3) & 4(50) \\ \text { CDI while not using PPI*, } \mathrm{n}(\%) & 2(16.7) & 4(50)\end{array}$

* PPI prescribed was pantoprazole

Table 4. Balance of key demographic and baseline clinical characteristics between two groups before and after propensity score adjustment.

\begin{tabular}{|l|c|c|}
\hline Key factors & $\begin{array}{c}\text { Before propensity } \\
\text { score adjustment }\end{array}$ & $\begin{array}{c}\text { After propensity score } \\
\text { adjustment }\end{array}$ \\
\hline PPI & 0.0088 & 0.3916 \\
\hline Gender & 0.2774 & 0.3779 \\
\hline Age & $<0.0001$ & 0.6239 \\
\hline Levofloxacin & 0.0346 & 0.5560 \\
\hline Ceftriaxone & 0.4068 & 0.7865 \\
\hline Ciprofloxacin & 0.0071 & 0.5037 \\
\hline Clindamycin & 0.6139 & 0.9489 \\
\hline GI disorder & 0.7108 & 0.8317 \\
\hline Malignancy & 0.1828 & 0.0475 \\
\hline SCr & 0.8068 & 0.7684 \\
Albumin & 0.8967 & 0.7533 \\
\hline
\end{tabular}

stool specimen was submitted to our hospital's microbiology laboratory. Our records also only indicated inpatient utilization of antibiotics, probiotics, and PPIs, with the understanding that controlling for confounding outpatient adherence as well as the changes made at outpatient setting would be difficult to analyze and interpret appropriately. Additionally, requiring included patients to have received a minimum of five days of high-risk antibiotic therapy may have excluded some patients who had CDI after receiving those high-risk antibiotic agents for short courses of empiric therapy. However, since the intent of this study was to evaluate the impact of probiotics for patients who received high-risk antibiotics for the treatment of documented infections, we decided to exclude patients who did not receive high-risk antibiotics for less than 5 days. Including patients who received shorter courses of multiple, high-risk antibiotic would have made our results more difficult to analyze and interpret. During the six months included in the chart review, no patient was identified to have received either carbapenem investigated (imipenem-cilastin, or meropenem) for five days or more without a history of CDI. The use of all carbapenems was restricted to infectious disease service as well as institutional antimicrobial stewardship team. Imipenem-cilastin and meropenem were often used empirically for patients who had a history of multiple drug resistant bacteria and then deescalated to ertapenem if there was no evidence of Pseudomonas infection. Concomitant use of antibiotics other than high-risk antibiotics before streamlining should be evaluated in the future study. Since FQs may carry higher risk of CDI than 3rd generation cephalosporins and carbapenems, the findings of this study could potentially be generalized to the broader population due to the aforementioned clinical practices.

\section{Conclusion}

This study showed that patients receiving high-risk antibiotics along with probiotics actually had a higher incidence of CDI than those who did not receive a probiotic. The difference did not achieve statistical significance once the propensity score was applied to remove the potential bias. Our findings based on adjusted odds ratio do not support the recommendation of the routine use of probiotics for the prevention of $\mathrm{CDI}$ among patients receiving high-risk antibiotics. With the recent outbreak and emergence of the BI/NAP1 highly-resistant strain of $C$. difficile, it is more important than ever to identify risk factors for $\mathrm{CDI}$ as well as avoidable causes of $\mathrm{CDI}$ such as PPIs and unnecessary use of antibiotics. Additional research with larger patient populations, uniform choice of probiotics and inclusion of broader classes of antimicrobial therapy are needed. Studies also should be powered to evaluate endpoints such as morbidity and mortality. In the meantime, alternative strategies should be applied to prevent CDI other than the use of prophylactic probiotics.

\section{Acknowledgement}

Justine Dickson, PharmD, BCACP and Whitney Y. Hung, PharmD, BCPS (AQ-ID) had full access to all of the data in the study and take responsibility for the integrity of the data and the accuracy of the data analysis. None of the authors have any financial relationships, interests, activities, or affiliations to disclose. 


\section{References}

1 Magill SS, Edward JR, Bamberg W, Zintars G Beldavs, Ghinwa Dumyati, et al. (2014) Multistate Point-Prevalence Survey of Health Care-Associated Infection. N Engl J Med 370: 1198-1208.

2 Heider F, Raza N, Komar M, Rahman O, Sartorius J, et al. (2012) Proton Pump Inhibitor Use Elevates the Risk of Severe Clostridium difficile Colitis. J Gastroenterol Hepatol Res 1: 53-56.

3 Vital Signs: Preventing Clostridium difficile Infection (2012) 61: 157-162.

4 National Healthcare Safety Network (NHSN) (2011) Facility-wide Inpatient Hospital-onset Clostridium difficile Infection (CDI) Outcome Measure.

5 U.S. Food and Drug Administration (FDA) (2012) FDA Medwatch Drug Safety Communication: Clostridium difficile-Associated Diarrhea can be Associated with Stomach Acid Drugs Known as Proton Pump Inhibitors (PPIs).

6 Johnston BC, Ma SS, Goldenberg JZ, Thorlund K, Vandvik PO, et al. (2012) Probiotics for the Prevention of Clostridium difficileAssociated Diarrhea: a Systematic Review and Meta-Analysis. Ann Intern Med 157: 878-888.

7 Allen SJ, Wareham K, Wang D, Bradley C, Hutchings H, et al. (2013) Lactobacilli and Bifidobacteria in the Prevention of AntibioticAssociated Diarrhoea and Clostridium difficile Diarrhoea in Older Inpatients (PLACIDE): A Randomised, Double-Blind, PlaceboControlled, Multicentre Trial. Lancet 382: 1249-1257.

8 Cohen SH, Gerding DN, Johnson S, Kelly CP, Loo VG, et al. (2010) Clinical Practice Guidelines for Clostridium difficile Infection in Adults: 2010 Update by the Society for Health Care Epidemiology of America (SHEA) and the Infectious Diseases Society of America (IDSA). Infect Control Hosp Epidemiol 31: 431-455.

9 Baxter R, Ray GT, Fireman BH (2008) Case-Control Study of Antibiotic Use and Subsequent Clostridium difficile - Associated Diarrhea in Hospitalized Patients. Infect Control Hosp Epidemiol 29: 44-50.

10 Pepin J, Saheb N, Coulombe MA, Alary ME, Corriveau MP, et al. (2005) Emergence of Fluoroquinolone as the Predominant Risk Factor for Clostridium difficile-Associated Diarrhea: A Cohort Study During an Epidemic in Quebec. Clin Infect Dis 41: 1254-1260.

11 Brown KA, Khanafer N, Daneman N, Fisman DN, (2013) Meta-Anyalsis of Antibiotics and the Risk of Community-Associated Clostridium difficile Infection. Antimicrob Agents Chemother 57: 2326-2332

12 Hickson, M (2011) Probiotics in the Prevention of AntibioticAssociated Diarrhea and Clostridium difficile infection. Therap Adv Gastroenterol 4:185-197.

13 Kotowska M, Albrecht P. Szajewska H (2005) Saccharomyces boulardii in the prevention of antibiotic-assocaited diarrhea in children: a randomized double-blind placebo-controlled trail. Aliment Pharmacol Ther 21: 583-590.

14 Can M, Besirbellioqlu BA, Avci IY, Beker CM, Pahsa A (2006) Prophylactic Saccharomyces boulardii in the prevention of antibioticassociated diarrhea: a prospective study. Med Sci Monit 12: 19-22.

15 Surawicz CM, Elmer GW, Speelman P, McFarland LV, Chinn J, et al. (1989) Prevention of antibiotic-associated diarrhea by Saccharomyces boulardii: a prospective study. Gastroenterology 96: 981-988.
16 McFarland LV, Surawicz CM, Greenberg RN, Elmer GW, Moyer KA, et al. (1995) Prevention of beta-lactam-associated diarrhea by Saccharomyces boulardii compared with placebo. Am J Gastroenterol 90: 439-48.

17 Shim JO (2014) Clostridium difficile in Children: To Treat or Not to Treat. Pediatr Gastroenterol Hepatol Nutr 17: 80-84.

18 Belanger SD, Boissinot M, Clairoux N, François J Picard, Michel G Bergeron, et al. (2003) Rapid detection of Clostridium difficile in feces by real-time PCR. J Clin Microbiol 41: 730-734.

19 Chitnis AS, Holzbauer SM, Belflower RM, Winston LG, Bamberg WM, et al. (2013) Epidemiology of Community-Associated Clostridium difficile Infection, 2009 through 2011. JAMA Intern Med 173: 13591367.

20 Adams DA, Riggs MM, Donskey CJ (2007) Effect of Fluoroquinolone Treatment on Growth of and Toxin Production by Epidemic and Nonepidemic Clostridium difficile Strains in the Cecal Contents of Mice. Antimicrob Agents Chemother 51: 2674-2678.

21 Sachs G, Shin JM, Briving C, Wallmark B, Hersey S, et al. (1995) The Pharmacology of the Gastric Acid Pump: the H+,K+ ATPase. Annu Rev Pharmacol Toxicol 35: 277-305.

22 Bavishi C, DuPont H L, (2011) Systematic Review: the Use of Proton Pump Inhibitors and Increased Susceptibility to Enteric Infection. Aliment Pharmacol Ther 34: 1269-1281.

23 McCarthy DM (2012) Proton Pump Inhibitor Use and Clostridium difficile colitis; Cause or Coincidence? J Clin Gastroenterol 46: 350-353.

24 Rao A, Jump RL, Pultz NJ, Pultz NJ, Donskey CJ (2006) In vitro Killing of Nosocomial Pathogens by Acid and Acidified Nitrite. Antimicrob Agents Chemother 50: 3901-3904.

25 Moshkowitz M, Ben-Baruch E, Kline Z, Shimoni Z, Niven M, et al. (2007) Risk Factors for Severity and Relapse of Pseudomembranous Colitis in an Elderly Population. Colorectal Dis 9: 173-177.

26 Rubin MS, Bodenstein LE, Kent KC (1995) Severe Clostidium difficile colitis. Dis Colon Rectum 38: 350-354.

27 Pépin J, Valiquette L, Alary ME, Villemure P, Pelletier A, et al. (2004) Clostridium difficile-Associated Diarrhea in a Region of Quebec from 1991 to 2003: a Changing Pattern of Disease Severity. CMAJ. 171: 466-472.

28 Del Piano M, Anderloni A, Balzarini M, Ballarè M, Carmagnola S, et al. (2012) The innovative potential of Lactobacillus rhamnosus LR06, Lactobacillus pentosus LPS01, Lactobacillus plantarum LP01, and Lactobacillus delbrueckii Subsp. delbrueckii LDD01 to restore the "gastric barrier effect" in patients chronically treated with PPI: a pilot study. J Clin Gastroenterol 46: S18-26.

29 Cannon JP, Lee TA, Bolanos JT, Danziger LH (2005) Pathogenic Relevance of Lactobacillus: a Retrospective Review of Over 200 cases. Eur J Clin Microbiol Infect Dis 24: 31-40.

30 Liong MT (2006) Safety of Probiotics: Translocation and Infection. Nutrition Reviews ${ }^{\circledR}$ 66: 192-202.

31 Flatley EA, Wilde AM, Nailor MD (2015) Saccharomyces boulardi for the Prevention of Hospital Onset Clostridium difficile Infection. J Gastrointestin Liver Dis 24: 21-24. 